\title{
Reciprocal Relationships between General (Propofol) Anesthesia and Circadian Time in Rats
}

\author{
Etienne Challet ${ }^{1,4}$, Sylviane Gourmelen', Paul Pevet', Philippe Oberling ${ }^{2}$ and Laure Pain*,3,4 \\ 'Department of Neurobiology of Rhythms, Institute of Cellular and Integrative Neurosciences, CNRS (UMR7 I 68), University L. Pasteur, \\ Strasbourg, France; 'Laboratory of Behavioural and Cognitive Neuroscience, CNRS (FRE2855), University L. Pasteur, Strasbourg, France; \\ ${ }^{3}$ INSERM U666 (GRERCA) and Hôpitaux Universitaires de Strasbourg, Faculty of Medicine, Strasbourg, France
}

\begin{abstract}
Several common postdischarge symptoms, such as sleep disorders, headache, drowsiness or general malaise, evoke disturbances of circadian rhythms due to jet lag (ie crossing time zones) or shift work rotation. Considering that general anesthesia is associated with numerous effects on the central nervous system, we hypothesized that it may also act on the circadian timing system. We first determined the effects of the circadian timing on general anesthesia. We observed that identical doses of propofol showed marked circadian fluctuations in duration of effects, with a peak at the middle of the resting period (ie $7 \mathrm{~h}$ after lights on). Then, we examined the effects of general anesthesia on circadian timing, by analysing stable free-running circadian rhythms (ie in constant environmental conditions), an experimental approach used widely in circadian biology. Free-running rats were housed in constant darkness and temperature to assess possible phase-shifting effects of propofol anesthesia according to the time of the day. When administered around $( \pm 2 \mathrm{~h})$ the daily rest/activity transition point, a 30-min propofol anesthesia induced a I-h phase advance in the free-running rest-activity rhythm, while anesthesia had no significant resetting effect at other times of the day. Anesthesia-induced hypothermia was not correlated with the phase-shifting effects of propofol anesthesia. From our results, anesthesia itself can reset circadian timing, and acts as a synchronizing cue for the circadian clock.

Neuropsychopharmacology (2007) 32, 728-735. doi:I0.I038/sj.npp. I30108I; published online 12 April 2006
\end{abstract}

Keywords: anesthetic; chronobiology; chronobiotic; propofol; circadian rhythm

\section{INTRODUCTION}

Biological rhythmicity is a fundamental regulatory principle in living organisms. Circadian rhythms (ie temporal variations within a period close to $24 \mathrm{~h}$ ) are controlled by the brain. This includes a main circadian clock, input pathways (synchronizers) connecting the clock to the external environment, and outputs by which the clock confers rhythmicity to most biochemical, physiological and behavioral variables of the organism (Turek et al, 1995). In mammals, the main circadian clock is located in the suprachiasmatic nucleus of the hypothalamus, and the main environmental synchronizer is light. Circadian rhythmicity can be disturbed not only by socioenvironmental factors, such as jet lag (ie crossing time zones) and

*Correspondence: Dr L Pain, GRERCA, Unité 666 INSERM, Faculté de Médecine, II rue Humann, 67000 Strasbourg, France, Tel: + 33390 2432 37/33 685436300, Fax: + 333902432 56,

E-mail: Laurepain@aol.com

${ }^{4}$ These authors contributed equally to the experiments.

Received 28 September 2005; revised I 5 February 2006; accepted 28 February 2006

Online publication: 13 March 2006 at http://www.acnp.org/citations/ Npp03 $1306050600 /$ default.pdf shift work rotation, but also by drugs acting on the central nervous system (so-called chronobiotic drugs).

Previous lines of research have found changes in toxic doses and duration of action of different general anesthetics depending of the time-of-day of administration, but it is still unclear to what extent such phenomena are directly under the control of circadian clocks (Chassard and Bruguerolle, 2004). Conversely, general anesthesia is a particular wakesleep state that is thought to alter circadian rhythms. Some studies suggest that general anesthetics disturb the circadian rhythms in the days following. The induction of ether or ketamine anesthesia during the resting phase (light period) of rodents has been shown to alter the daily rhythms of heart rate, body temperature and locomotor activity in the days following (Prudian et al, 1997). In humans, anesthesia and surgery with operative stress delay the onset of nocturnal melatonin secretion during the first postsurgical evening, suggesting a phase shift in the circadian secretion of melatonin (Guo et al, 2002; Karkela et al, 2002; Reber et al, 1998). Such alterations, however, have been observed when anesthesia was performed in conjunction with surgery. The role of surgery itself cannot be fully excluded (for discussion, see eg Karkela et al, 2002). Finally, from all these studies, it is still unclear if general anesthesia per se has a direct effect on brain regulation of circadian timing. 
The aim of the study was to examine the connection between circadian timing and general anesthesia. For this, we used propofol, a general anesthetic commonly used for induction and maintenance of general anesthesia for short as well as long procedures. As a result of its pharmacokinetic parameters (short half-life elimination, rapid emergence from anesthesia), the use of propofol is growing, particularly in ambulatory practice, which allows the patient to return home the same day (Gupta, 2004). Any disturbance of circadian rhythms, such as jet lag, may be responsible for a variety of medical symptoms that have been already described after general anesthesia, including sleep disorders, drowsiness, and mood alteration (Deutsch and $\mathrm{Wu}, 2003)$. Therefore, it is clinically relevant to determine whether short-duration propofol anesthesia has any deleterious impact on circadian rhythms.

In the present study, to further understand possible relations between anesthesia and the circadian system, we investigated two related aspects: firstly, the effects of timeof-day on propofol-induced anesthesia; secondly, the effects of short-duration propofol anesthesia on the circadian clock. To assess the direct action of anesthesia on the circadian system, we examined its effects on stable freerunning circadian rhythms in animals exposed to constant conditions (ie in environmental conditions with no external cues signaling time-of-day).

\section{MATERIALS AND METHODS}

\section{Animals}

For experiment 1, 36 male Long Evans rats (Janvier, Le Genest-St-Isle, France) weighing 200-230 g at the beginning of the experiment were housed two per cage in a temperature-controlled room of standard housing $\left(23 \pm 1^{\circ} \mathrm{C}\right)$. For experiment 2, 12 male Long Evans rats weighing 200-230 $\mathrm{g}$ at the beginning of the experiment were individually housed in cages equipped with a running wheel ( $9 \mathrm{~cm}$ wide $\times 34.5 \mathrm{~cm}$ diameter) in a temperature controlled room $\left(23 \pm 1^{\circ} \mathrm{C}\right)$. For experiment 3, 16 male Long Evans rats weighing $140-160 \mathrm{~g}$ at the time of surgery were used to take into account the two additional weeks of recovery from surgery and habituation to the perfusion device. These rats were housed in cages with a running wheel as described for experiment 2. During all the experimental procedures, the animals were kept isolated from environmental stimuli. Food and water were available ad libitum, and a fan provided constant fresh air flow as well as constant background noise.

All experimental procedures were carried out in accordance with the National Council Directive (\#87848, October 19, 1989, Ministère de l'Agriculture et de la Forêt, Service Vétérinaire de la Santé et de la Protection Animales) and the European Communities Council Directive (\#86/609/EEC, November 24, 1987). All efforts were made to minimize the number of animals used (repeated measures in experiments 2 and 3 ) and any potential suffering.

\section{Drugs}

Propofol (10 mg/ml Diprivan; Zeneca, London, UK) was injected intraperitoneally (i.p.) at the dose of $100 \mathrm{mg} / \mathrm{kg}$ in experiments 1 and 2, and 30 min-infused i.p. at the dose of $60 \mathrm{mg} / \mathrm{kg}$ in experiment 3. Intralipids $10 \%$ was used as a control lipidic solution (Fresenlus Kabl, Sèvres, France). The dose and regimen of propofol administration have been determined in previous experiments using rats weighing $160-180 \mathrm{~g}$, in which the anesthetic effect was determined by the loss of righting reflex (Pain et al, 1996, 1997).

\section{Procedures}

Experiment 1. The animals were maintained under a $12 \mathrm{~h}$ light-12 h dark cycle for 2 weeks to habituate them to the experimental conditions (light, 0700-1900). Propofol $(100 \mathrm{mg} / \mathrm{kg})$ was administered at six local times: 0600, $1000,1400,1800,2200$, and 0200 ( $n=6$ per time point).

Following treatment, rats were placed on their back every minute to determine the onset for loss of righting reflex (inability of the animal to right itself on all four paws when positioned on its back). The duration of loss of righting reflex (LRR) was defined as the time interval between LRR until return of the reflex, the rat having been placed on its back and turned to a prone position two consecutive times (Rebuelto et al, 2004).

\section{Experiment 2.}

Surgery: Under isoflurane anesthesia using local anesthesia as needed (lidocaine), each animal was implanted i.p. with a Minimitter transmitter (Mini-mitter Inc., Sun river, USA) to record general locomotor activity and body temperature.

Experimental design: After 1 week to the exposure of a $12 \mathrm{~h}$ light-12 $\mathrm{h}$ dark cycle, the animals were transferred to continuous dim red light conditions (darkness for rats) during 2 weeks before beginning the experiment. Wheelrunning, body temperature and general locomotor activity data were continuously recorded and the data were stored in 5 min time bins by an acquisition system (Mini-mitter). To investigate possible shifting effects of short-lasting propofol anesthesia, rats were infused with propofol $(100 \mathrm{mg} / \mathrm{kg})$ at two times of the daily cycle (ie activity onset and offset). Note that for nocturnal animals such as rats, circadian time (CT) 12 and CT0 define the time of activity onset and offset, respectively. According to this nomenclature, anesthesia was induced at CT12 and CT0. Each animal received two infusions using either drug or vehicle (lipid) separated by 10 days ( $n=6$ per condition). This preliminary experiment was set to determine whether anesthesia induced by a bolus of propofol may elicit phase shifts.

\section{Experiment 3.}

Surgery: To avoid any potential effects of handling for the infusion of propofol or vehicle, animals were implanted with an intraabdominal catheter under isoflurane anesthesia using local anesthesia as needed (lidocaine). The distal end of the catheter was placed on a swivel fixed to a bar hung from the top of the cage. This method has been previously described, except that the catheter was implanted subcutaneously (Pitrosky et al, 1999). The swivel was connected to a syringe carried by a pump (Harvard Apparatus, Holliston, MA, USA) controlled by an electronic timer. Concomitant with catheter setting, each animal was 
implanted i.p. with a Minimitter transmitter as above to record general locomotor activity and body temperature.

Experimental design: Before the start of the experiment, the implanted animals were maintained under a $12 \mathrm{~h}$ light-12 h dark cycle for 2 weeks to habituate them to the experimental situations. Thereafter, the animals were transferred to continuous dim red light conditions during at least 2 weeks before beginning the experiment and throughout experimental duration. Wheel-running, body temperature and general locomotor activity data were continuously recorded and the data were stored in $5 \mathrm{~min}$ time bins by an acquisition system (Mini-mitter). To assess in detail the chronobiotic effects of short-lasting propofol anesthesia, rats were perfused with propofol $(60 \mathrm{mg} / \mathrm{kg})$ infused continuously during $30 \mathrm{~min}$ at various times of the daily cycle (ie early morning, midday, late afternoon, early night, midnight, and late night). Using the same nomenclature as above, anesthesia was induced at CT2, CT6, CT10, CT14, CT18, and CT22. Control animals received a perfusion of vehicle (lipid) solution. Each animal received several perfusions (with a maximum of five) using either drug or vehicle separated by at least 10 days, for as long as they maintained stable free-running rhythms. No animal received more than one treatment at a given time. This experiment allowed us to construct a phase response curve to propofol anesthesia by plotting the elicited phase shifts as a function of circadian time of treatment.

\section{Data Analysis}

Determination of the daily onsets of locomotor activity and daily increases of body temperature were performed with ClockLab (Actimetrics, Evanston, USA). The magnitude and direction of the elicited phase shifts were estimated by linear regressions fitted to the onsets, considering eight cycles before and eight cycles after treatment, excluding the first two cycles following treatment. The phase shift was calculated as the difference between the fitted lines (onsets) before and after treatment.

Acute changes in body temperature were determined as the difference between the mean value of endogenous abdominal temperature during $1 \mathrm{~h}$ before the perfusion and the lowest value during the $4 \mathrm{~h}$ following the onset of perfusion (SigmaPlot, Jandel Scientific, San Rafael, CA, USA).

\section{Statistical Analysis}

Experiment 1. Statistical analyses of data were performed using one way analyses of variance (ANOVA) (dependent factors: onset of LRR, duration of LRR; between factor: time). When differences between the various times of testing were detected, data were further analysed by the cosinor method (Nelson et al, 1979) to explore circadian rhythmicity. If substantiated, we determined the mesor (a 24-h rhythm adjusted mean), amplitude (one-half the peak trough variation due to rhythmicity), and acrophase (peak-time) as derived by the least-squares approximation of the time series data by the best fitting $24 \mathrm{~h}$ cosine curve.
Experiment 2. Statistical analyses of data were performed using one way ANOVA (dependent factors: phase shift of locomotor activity, phase shift of temperature, between factors: Intralipids at CT0, Intralipids at CT0, propofol at CT0, propofol at CT12), followed by a post hoc bonferonni $t$-test.

Experiment 3. Statistical analyses of data were performed using successive ANOVA for repeated measures with the following variables: two dependent, phase shift and temperature change, and three independent variables, treatment (propofol anesthesia $v s$ intralipids injection), circadian time (CT2, CT6, CT10, CT14, CT18, or CT22) and order of treatment $(1,2,3$, or 4 th). Levene's tests were systematically performed to test for equality of variance (BMDP Software, Dixon, California University). The level of statistical significance was set at $p<0.05$. Post hoc analyses were performed using $t$-test with Bonferonni correction for multiple comparisons.

\section{RESULTS}

\section{Experiment 1}

Significant temporal differences were detected in the duration of LRR. ANOVA revealed an overall significant effect of time $(\mathrm{F}(5,31)=33.38 ; p<0.0001)$. The longest pharmacological response occurred in rats injected during the light (rest period) phase and the shortest response in the dark (activity period) phase. A significant circadian rhythm of the duration of LRR was observed using single cosinor analysis with an acrophase at $1410 \mathrm{~h} \pm 26 \mathrm{~min}$ (ie $7 \mathrm{~h}$ after light on; Figure 1). No time-dependent differences were detected in the onset of LRR. $(F(5,31)=0.70$; NS). Mean $( \pm$ SD) onsets of LRR were 9.8 $( \pm 1.5), 9.3( \pm 2.0), 10.3$ $( \pm 0.8), 8.83( \pm 1.3), 9.0( \pm 2.2)$, and $9.7( \pm 1.6) \mathrm{min}$. for the six local times: $0600,1000,1400,1800,2200$, and 0200 , respectively.

\section{Experiment 2}

ANOVA detected a significant effect of treatment on both the phase shift of locomotor activity rhythm and the phase

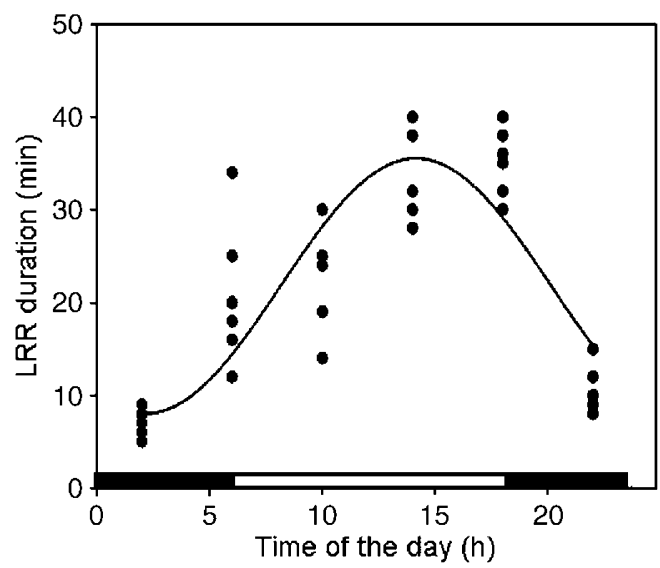

Figure I Duration of the loss of righting reflex (LRR) following propofol injection $(100 \mathrm{mg} / \mathrm{kg})$ in rats at different times of the day. 
shift of temperature (locomotor activity, $\mathrm{F}(3,20)=4.48$; $p<0.015$ and temperature, $\mathrm{F}(3,20)=9.30 ; p<0.0001)$. Post hoc analysis showed that propofol anesthesia led to larger phase shifts of both locomotor activity and temperature than those induced by intralipids injection when administered at circadian time CT12, but not at CT0 (Figure 2a). The amplitude of the shifts for activity and temperature rhythms were highly correlated $\left(r^{2}=0.81\right.$; Figure $\left.2 \mathrm{~b}\right)$.

\section{Experiment 3}

Phase shifts of rest-activity rhythm. Propofol treatment led to a subsequent phase-advance in the circadian rhythm of rest-activity that was not detected following vehicle perfusion (Figure 3). One-way ANOVA showed an overall significant effect of treatment (propofol anesthesia $v s$ control) on the phase shift $(\mathrm{F}(1,73)=5.52 ; p=0.02)$. There was no effect of the order of treatment $(\mathrm{F}(3,67)=2.67$; $p=0.44)$ and the interaction between the treatment and the order of treatment was not significant (treatment $\times$ order: $\mathrm{F}(3,67)=0.55 ; p=0.65)$. A two-way ANOVA showed a
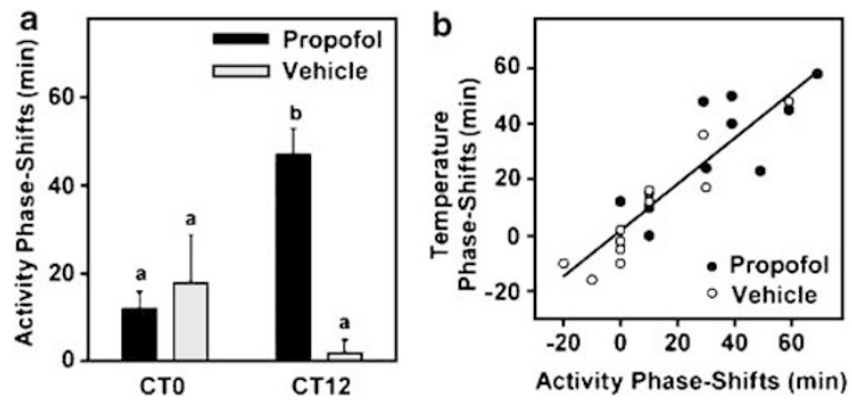

Figure 2 (a) Phase-shifts in circadian rhythm of rest-activity in rats injected with a bolus of propofol $(100 \mathrm{mg} / \mathrm{kg}$ ) or vehicle (intralipids solution) at two time points of the daily cycle. Positive values are phase advances. Values are means \pm SEM ( $n=6$ per group). Differences according to the circadian time (CT) are indicated by groups having different letters $(p<0.05)$. (b) Positive correlation between the shifts in circadian rhythms of rest-activity and body temperature in rats injected with a bolus of propofol ( $100 \mathrm{mg} / \mathrm{kg}$ ) or vehicle (intralipids solution). Positive and negative values are phase advances and delays, respectively. Data were pooled from circadian times 0 and 12. To draw the regression line $\left(r^{2}=0.81\right)$, data were fitted to the following equation: $(y=A x+B)$.
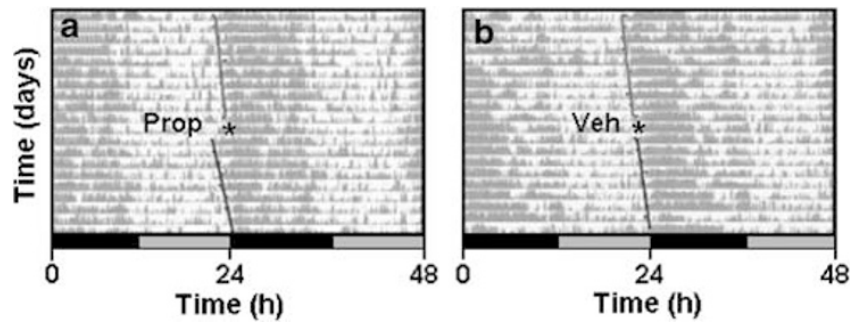

Figure 3 General locomotor activity (arbitrary units) in rats housed in constant darkness. Successive 24-h periods are double-plotted (48-h horizontal time scale). At circadian time 14, animals in (a and b) were perfused via implanted catheter with propofol $(60 \mathrm{mg} / \mathrm{kg}$ ) and vehicle solution of intralipids, respectively. Note that propofol treatment leads to a subsequent phase-advance in the circadian rhythm of rest-activity, that is not detected after perfusion of the vehicle. Onset of perfusion is indicated by an asterisk. significant effect of circadian time $(\mathrm{F}(5,63)=3.03 ; p=0.02)$ and treatment $(\mathrm{F}(1,63)=8.88 ; p<0.01)$ on the phase shift and a significant interaction between circadian time and treatment $(\mathrm{F}(5,63)=7.12 ; p<0.0001)$. Post hoc analysis showed that significant differences between propofol anesthesia and intralipids treatment groups were observed only at circadian times CT10 and CT14 (all $p<0.0001$; Figure 4). As the shifting effects of propofol anesthesia were found to be similar for the circadian rhythms of both restactivity and body temperature in experiment 2 (see above), the latter were not quantified in experiment 3.

Acute changes in body temperature. Anesthesia-induced hypothermia depended on circadian time with the largest magnitude at the sleep/wake transition (Figures 5 and 6). Two-way ANOVA with repeated measures (between factors: circadian time, treatment; within factor: temperature change)

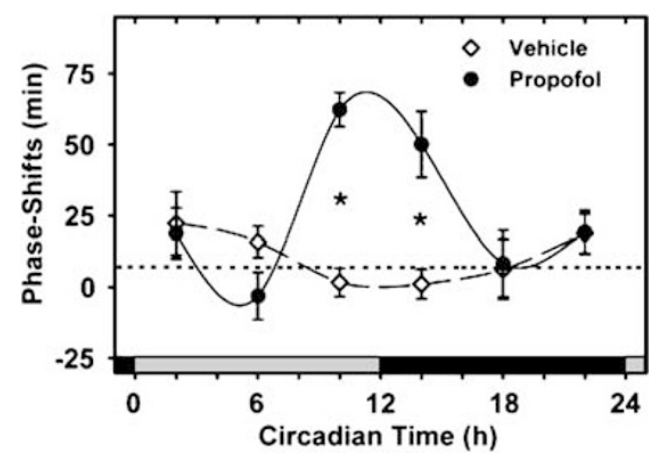

Figure 4 Phase-shifts in circadian rhythm of rest-activity of rats perfused via implanted catheter with propofol $(60 \mathrm{mg} / \mathrm{kg})$ or vehicle (intralipids solution) at six time points over the daily cycle. Positive and negative values are phase advances and delays, respectively. Values are means \pm SEM. * $p<0.05$.
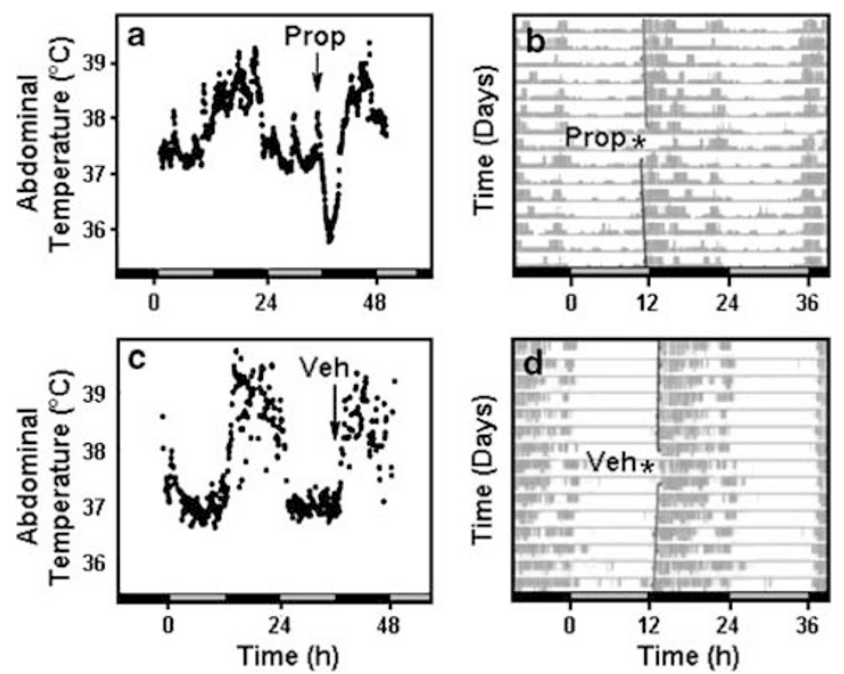

Figure 5 (a and c) 5-min recording of abdominal temperature before and after a 30-min perfusion with propofol or vehicle (intralipids solution) started at circadian time 10 in rats housed in constant darkness. (b and d) Show data of ( $a$ and $c$ ) respectively, with temperature plotted only when it was up to $37^{\circ} \mathrm{C}$ to facilitate visualization of a phase-advance after propofol treatment (b), but not after intralipids administration (d). Arrows (a and c) and asteriks ( $b$ and $d$ ) indicate the onset of perfusion. 


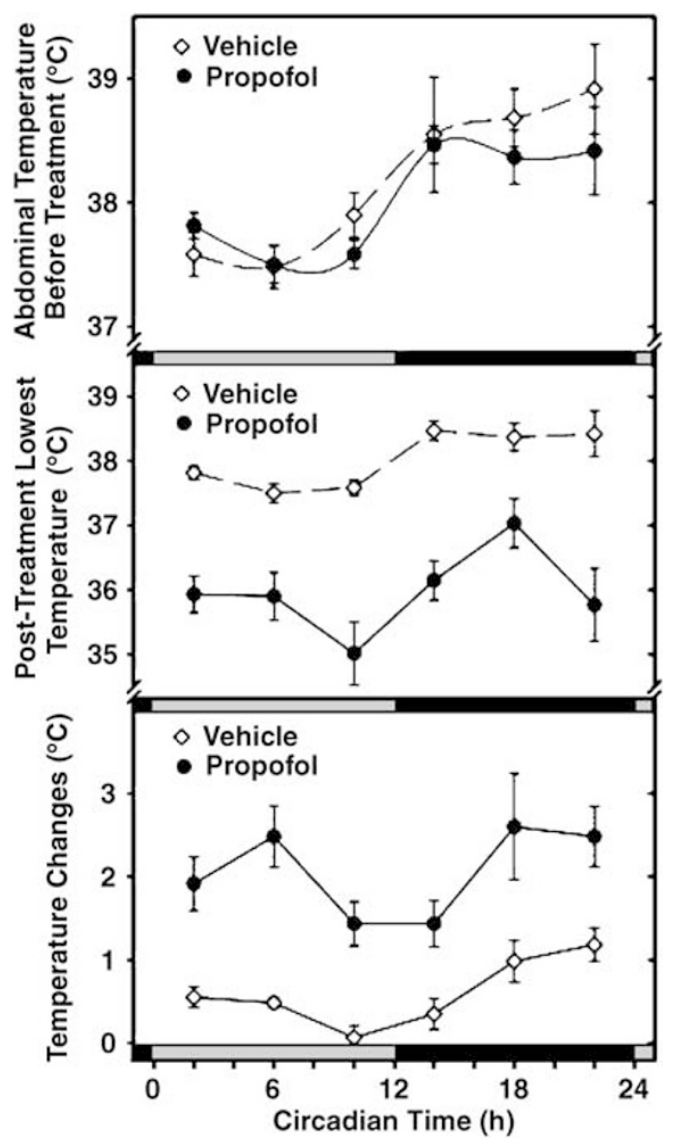

Figure 6 Upper panel: mean abdominal temperature before the perfusion (propofol or vehicle solution of intralipids) displays a circadian rhythm in rats housed in constant darkness, with low values during the resting period (ie subjective day, corresponding to the interval from circadian time 0 to circadian time 12) and high values during the active period (ie subjective night, corresponding to the interval from circadian time 12 to circadian time 24 which is equivalent to circadian time 0 ). Middle panel: effect of the 30-min perfusion with propofol or vehicle solution of intralipids on abdominal temperature. There was a clear hypothermia resulting from propofol treatment for all time points studied. Lower panel: changes in internal temperature after perfusion of propofol or vehicle assessed as the temperature difference before and after treatment (ie difference between data showed in upper and middle panels, respectively). Values are means \pm SEM.

showed a significant effect of treatment $(\mathrm{F}(1,63)=30.41$; $p<0.0001)$ and circadian time $(\mathrm{F}(5,63)=3.03 ; p=0.02)$ on temperature change. There were significant interactions between treatment and temperature change $(\mathrm{F}(1,63)=$ $74.25 ; p<0.0001)$, and between circadian time and temperature change $(F(5,63)=4.84 ; p<0.001)$, but no significant interaction was detected between treatment, circadian time and temperature change $(\mathrm{F}(5,63)=0.53 ; p=0.76)$.

\section{DISCUSSION}

The present study clearly demonstrates a reciprocal interaction between circadian timing and general anesthesia. The duration of anesthesia induced by a given dose of propofol showed marked circadian fluctuations (experiment 1) whereas propofol anesthesia per se had a direct resetting effect on circadian timing (experiments 2 and 3 ).

\section{Chronopharmacology of Propofol Anesthesia}

We demonstrate for the first time that the duration of propofol anesthesia exhibits a statistically significant circadian rhythm with a three-fold amplitude. Our results show that the median duration of propofol anesthesia is longest when rats are injected during the light (rest period) phase at 1400 and 1800 and shortest when injected during the dark (activity period) phase at 2200 and 0200. Our results agree with those of preceding studies showing that the anesthetic effects of other agents, such as althesin, pentobarbital, ketamine, and halothane, are higher during the natural sleep period (Bruguerolle et al, 1977; Giedt et al, 1978; Nelson and Halberg, 1973). Recent experiments in rodents also showed that the duration of Ketamine effects varies over $24 \mathrm{~h}$ (Rebuelto et al, 2004; Sato et al, 2004).

We did not measure blood levels of propofol in this study. It is thus possible that our observations may result partly from circadian fluctuation of pharmacokinetic properties. Propofol is mainly metabolized by hepatic microsomal systems (Court et al, 2001). Circadian fluctuations have been reported in rat hepatic enzyme activity with high values in the dark (activity) phase and low values in the light (resting) phase (Furukawa et al, 1999). Time of daydependent differences in the duration of propofol anesthesia may theoretically be due to circadian rhythms in hepatic metabolism of this drug. We believe that such effects are unlikely to fully explain our findings. As ketamine is mainly metabolized by the hepatic microsomal system (cytochrome $\mathrm{P} 450 \mathrm{~s}$ ), a previous study investigated daily variation of ketamine effects as a function of circadian fluctuations in pharmacokinetics. No significant time-dependent differences in plasma concentrations of ketamine between activity and resting periods in mice were observed, in contrast to the time-of-day differences in the duration of ketamine anesthesia. In that study, the results observed using NMDA receptor epsilon1 subunit KO mice (ie absence of daily variations in ketamine effects, ketamine constituting an NMDA antagonist) suggest rather that daily variations in anesthetic effect depend on circadian differences in the sensitivity of the central nervous system to the drug (Sato et al, 2004). Propofol is supposed to act mainly via $\mathrm{GABA}_{\mathrm{A}}$ receptors to induce anesthesia (Trapani et al, 2000). Daily variations in $\mathrm{GABA}_{\mathrm{A}}$ receptor function have been described in the cerebral cortex of hamster. Maximal GABA receptor-binding affinity and $\mathrm{GABA}_{\mathrm{A}}$ receptor postsynaptic activity are observed during the activity period (Kanterewicz et al, 1995). Moreover, we found that the duration of anesthesia is two-thirds higher during the natural sleep cycle of rats. Natural sleep and anesthesia appear to share common mechanisms (Nelson and Guo, 2002). Endogenous neuromodulators known to regulate sleep also alter anesthetic effects, and anesthetics induce sleep when directly injected into brain areas known to regulate sleep (Tung and Mendelson, 2004). Sleep deprivation potentiates the anesthetic potency of anesthetics, such as propofol, therefore, establishing a link between sleep need and anesthesia (Tung et al, 2002). Both sleep regulation and sleep need are characterized by a circadian pattern. It is thus possible that our observations may be partly the result of physiological interaction between propofol anesthesia and brain sleep regulation. From our 
data, we cannot characterize the precise mechanisms involved, but we speculate that time-of-day dependent differences in propofol anesthesia may be due to circadian variations in brain sensitivity to the anesthetic.

\section{Chronobiotic Effects of Propofol Anesthesia}

More importantly, we showed that propofol anesthesia in rats elicited $1-h$ phase advances of the activity-rest rhythm when administered around $( \pm 2 \mathrm{~h})$ the transition period from sleep to wakefulness, while it had no significant resetting effect at other times of the day. As the phaseshifting effects were (1) detected in constant environmental conditions, (2) remained stable over days, they suggest strongly that the changes are mediated by clock-dependent mechanisms. Comparable results were obtained after propofol was administered i.p. as a bolus or during 30 min through an implanted catheter, excluding any effect of handling. To our knowledge, this is the first demonstration that general anesthesia (propofol) has a chronobiotic effect. The amplitude of the phase shifts produced by brief anesthesia with perfused propofol is far from being negligible because it represents about half the maximal resetting effect of a light pulse in rodents housed in constant darkness (Takahashi et al, 1984).

One obvious change associated with general anesthesia is transient hypothermia. Changes in temperature can phaseshift circadian neuronal rhythms of suprachiasmatic nuclei maintained in vitro (Herzog and Huckfeldt, 2003; Ruby et al, 1999), raising the possibility that anesthesia-induced hypothermia plays an active role in the observed phaseshifts. Notably, hypothermia reached the lowest (absolute) values after propofol perfusion at the sleep/wake transition (ie at circadian time 10), the same time as propofol treatment induced subsequent phase-advances (Figures 3 and 5). However, at the next time point studied (ie at circadian time 14), this correlation was no longer tenable, suggesting that there is no causal relationship between hypothermia and phase-shifting properties. Moreover, when the influence of the endogenous rhythm of body temperature is dissociated (in this case, subtracted) from the acute effects of propofol administration, it appears that the phase-shifting effect of propofol is greater at times when propofol is least effective in producing hypothermia (ie at the transition from the resting to active period). In keeping with other studies showing marked effects of temperature changes on peripheral oscillators, but not on the suprachiasmatic clock (Brown et al, 2002), anesthesia-induced hypothermia by itself cannot explain the resetting properties of propofol anesthesia.

Short periods of immobilization can also produce phaseshifts (in this case, delays) in the activity-rest rhythm when induced during the early period of activity in (nocturnal) hamsters (Van Reeth et al, 1991). Thus, the lack of motor activity due to anesthesia during the early active period may theoretically play a role in the observed phase-shifts. Considering that phase-advances and delays are mediated by different transduction pathways (review in Gillette and Mitchell, 2002), this hypothesis is highly unlikely because the phase shifts elicited by propofol sedation are phaseadvances, and not phase-delays as described for immobilization itself.
From our results, anesthesia itself was responsible for resetting circadian timing, and acted as a synchronizing cue for the circadian clock. Based on their effects according to a temporal window of sensitivity, synchronizing cues can be divided into two classes: photic and nonphotic factors (Challet and Pevet, 2003). Photic cues (eg short exposure to light in darkness) lead to phase delays and advances during the early and late night, respectively, with low or no resetting effects during the day. Nonphotic cues (eg transient behavioral arousal associated with motor activity) studied in nocturnal mammals are capable of inducing phase-advances especially when applied during the middle of the day (ie the resting period in nocturnal species), with little or no resetting effects during the night (for review, see Challet and Pevet, 2003). Propofol anesthesia produces phase advances in the late subjective day, similar to treatment with exogenous melatonin (Pitrosky et al, 1999). As far as we are currently aware, besides general anesthesia the only other synchronizing cues which share the ability to produce circadian phase-advances in the early subjective night are dark pulses applied in constant light (Dwyer and Rosenwasser, 2000; Mendoza et al, 2004). This atypical circadian window of sensitivity, which does not match with the intracellular transduction pathways described for the suprachiasmatic clock (Gillette and Mitchell, 2002), awaits further investigation to better understand its underlying mechanisms.

\section{Clinical Significance}

Several clinical symptoms can occur in the days following general anesthesia, including sleep disorders, drowsiness and mood alteration (Wu et al, 2002). These symptoms are also commonly reported in jet-lagged travelers (Haimov and Arendt, 1999). We thus raised the hypothesis that general anesthesia may affect circadian timing similar to jet lag. Impaired synchronization to local time (so-called 'desynchronization'), as occurring after jet lag, is well known to be associated with sleep deprivation and altered vigilance leading to increased accidents and reduced work productivity, with obvious health and economic consequences.

Data from human studies have shown disturbances in the pattern of melatonin secretion following general anesthesia associated with surgery (Guo et al, 2002; Karkela et al, 2002; Reber et al, 1998). Secretion of melatonin by the pineal gland is commonly used as a circadian phase marker (Cassone et al, 1993; Pevet, 2003). Any alteration detected in the timing of circulating melatonin is therefore considered to reflect altered functioning of the suprachiamatic clock. However, in all these studies the role of surgery itself has not been excluded (for discussion, see eg Karkela et al, 2002). Using propofol, the present data supports our working hypothesis that general anesthesia per se alters daily temporal organization. As demonstrated here, propofol anesthesia in laboratory-controlled conditions has potent chronobiotic effects (ie phase resetting of the circadian clock). Therefore, its occurrence in real-life situations could lead to a temporarily impaired synchronization to local time, similar to that occurring during jet lag. From a clinical point of view, studying the chronobiotic effects of short-term propofol anesthesia appeared particularly relevant. Indeed, 
propofol is largely used in ambulatory surgery and/or short anesthesia for medical procedures (eg therapeutic radiology or digestive endoscopies). On the days following this type of short anesthesia, it is important to consider the quality of recovery at home in terms of health and economic factors. As mentioned above, any disturbance of the circadian rhythm may be responsible for a variety of medical symptoms, including sleep disorders, fatigue and mood alteration. Such symptoms appear to be quite common postdischarge symptoms even when short anesthesia is performed without surgery, for example, with regard to medical procedures (for a recent review, see $\mathrm{Wu}$ et al, 2002). In human volunteers, propofol anesthesia has been shown to disturb the subsequent sleep period (Ozone et al, 2000). Although other factors, such as release from acute stress and anticipatory anxiety about the procedure, might play a role in such symptoms, our current working hypothesis is that some of the postdischarge symptoms related to altered vigilance and sleep disturbances are the consequences of deleterious effects of general anesthesia on the circadian timing system. We thus propose to examine the consequences of the resetting effect of anesthesia on circadian timing in humans. This is currently under investigation in subjects exposed to regular synchronizing stimuli. If further data confirm our working hypothesis, preventive and/or therapeutic actions, such as timed exposure to light or appropriate chronobiotic drugs, could be used to counteract the rhythm disorders caused by general anesthesia and the associated consequences for patients.

Finally, we observed circadian rhythmicity of the duration of propofol anesthesia. These findings in rats together with previous data on the time of day-dependent effects of general anesthetics in animals, must be taken into account in pharmacological approaches to anesthesia in both human and non-human species.

\section{ACKNOWLEDGEMENTS}

We thank Dr David Hicks for helpful comments on the manuscript. This study was financially supported by a Program Research Award from the European Society of Anesthesiologists (LP), by a 'crédit exceptionnel pour jeunes équipes' from CNRS (EC) and 'ACI Plates-formes d'explorations fonctionnelles thématisées' from the Ministère délégué à la Recherche (PP).

\section{REFERENCES}

Brown SA, Zumbrunn G, Fleury-Olela F, Preitner N, Schibler U (2002). Rhythms of mammalian body temperature can sustain peripheral circadian clocks. Curr Biol 12: 1574-1583.

Bruguerolle B, Mesdjian E, Valli M, Blanc MC, Andanson P, Jadot $\mathrm{G}$ et al (1977). Acute toxicity of 5 intravenous anesthetics in rats depending on age and sex. Therapie 32: 375-380.

Cassone VM, Warren WS, Brooks DS, Lu J (1993). Melatonin, the pineal gland, and circadian rhythms. J Biol Rhythms 8(Suppl): S73-S81.

Challet E, Pevet P (2003). Interactions between photic and nonphotic stimuli to synchronize the master circadian clock in mammals. Front Biosci 8: s246-s257.
Chassard D, Bruguerolle B (2004). Chronobiology and anesthesia. Anesthesiology 100: 413-427.

Court MH, Duan SX, Hesse LM, Venkatakrishnan K, Greenblatt DJ (2001). Cytochrome P-450 2B6 is responsible for interindividual variability of propofol hydroxylation by human liver microsomes. Anesthesiology 94: 110-119.

Deutsch N, Wu CL (2003). Patient outcomes following ambulatory anesthesia. Anesthesiol Clin North America 21: 403-415.

Dwyer SM, Rosenwasser AM (2000). Effects of light intensity and restraint on dark-pulse-induced circadian phase shifting during subjective night in Syrian hamsters. J Biol Rhythms 15: 491-500.

Furukawa T, Manabe S, Watanabe T, Sharyo S, Mori Y (1999). Sex difference in the daily rhythm of hepatic P450 monooxygenase activities in rats is regulated by growth hormone release. Toxicol Appl Pharmacol 161: 219-224.

Giedt Jr WR, Lakin ML, Winters WD (1978). Diurnal response to ketamine and gamma-hydroxybutyrate and its possible relationship to pineal indoleamines. Neuropharmacology 17: 221-228.

Gillette MU, Mitchell JW (2002). Signaling in the suprachiasmatic nucleus: selectively responsive and integrative. Cell Tissue Res 309: 99-107.

Guo X, Kuzumi E, Charman SC, Vuylsteke A (2002). Perioperative melatonin secretion in patients undergoing coronary artery bypass grafting. Anesth Analg 94: 1085-1091 (Table of contents).

Gupta A (2004). Strategies for outpatient anaesthesia. Best Pract Res Clin Anaesthesiol 18: 675-692.

Haimov I, Arendt J (1999). The prevention and treatment of jet lag. Sleep Med Rev 3: 229-240.

Herzog ED, Huckfeldt RM (2003). Circadian entrainment to temperature, but not light, in the isolated suprachiasmatic nucleus. J Neurophysiol 90: 763-770.

Kanterewicz BI, Rosenstein RE, Golombek DA, Yannielli PC, Cardinali DP (1995). Daily variations in GABA receptor function in Syrian hamster cerebral cortex. Neurosci Lett 200: 211-213.

Karkela J, Vakkuri O, Kaukinen S, Huang WQ, Pasanen M (2002). The influence of anaesthesia and surgery on the circadian rhythm of melatonin. Acta Anaesthesiol Scand 46: 30-36.

Mendoza J, Dardente H, Escobar C, Pevet P, Challet E (2004). Dark pulse resetting of the suprachiasmatic clock in Syrian hamsters: behavioral phase-shifts and clock gene expression. Neuroscience 125: 529-537.

Nelson NP, Guo M (2002). The sedative component of anesthesia is mediated by $\mathrm{GABA}(\mathrm{A})$ receptors in an endogenous sleep pathway. Nature Neurosci 5: 979-984.

Nelson W, Halberg F (1973). An evaluation of time-dependent changes in susceptibility of mice to pentobarbital injection. Neuropharmacology 12: 509-524.

Nelson W, Tong YL, Lee JK, Halberg F (1979). Methods for cosinor-rhythmometry. Chronobiologia 6: 305-323.

Ozone M, Itoh H, Yamadera W, Ohbuchi K, Hayashida K, Sasaki M et al (2000). Changes in subjective sleepiness, subjective fatigue and nocturnal sleep after anaesthesia with propofol. Psychiatry Clin Neurosci 54: 317-318.

Pain L, Oberling P, Sandner G, Di Scala G (1996). Effect of propofol on affective state as assessed by place conditioning paradigm in rats. Anesthesiology 85: 121-128.

Pain L, Oberling P, Sandner G, Di Scala G (1997). Effect of midazolam on propofol-induced positive affective state assessed by place conditioning in rats. Anesthesiology 87: 935-943.

Pevet P (2003). Melatonin: from seasonal to circadian signal. J Neuroendocrinol 15: 422-426.

Pitrosky B, Kirsch R, Malan A, Mocaer E, Pevet P (1999). Organization of rat circadian rhythms during daily infusion of melatonin or S20098, a melatonin agonist. Am J Physiol 277: R812-R828.

Prudian F, Gantenbein M, Pelissier AL, Attolini L, Bruguerolle B (1997). Daily rhythms of heart rate, temperature and locomotor 
activity are modified by anaesthetics in rats: a telemetric study. Naunyn Schmiedebergs Arch Pharmacol 355: 774-778.

Reber A, Huber PR, Ummenhofer W, Gurtler CM, Zurschmiede C, Drewe J et al (1998). General anaesthesia for surgery can influence circulating melatonin during daylight hours. Acta Anaesthesiol Scand 42: 1050-1056.

Rebuelto M, Ambros L, Waxman S, Montoya L (2004). Chronobiological study of the pharmacological response of rats to combination ketamine-midazolam. Chronobiol Int 21: 591-600.

Ruby NF, Burns DE, Heller HC (1999). Circadian rhythms in the suprachiasmatic nucleus are temperature-compensated and phase-shifted by heat pulses in vitro. J Neurosci 19: 8630-8636.

Sato Y, Kobayashi E, Hakamata Y, Kobahashi M, Wainai T, Murayama $\mathrm{T}$ et al (2004). Chronopharmacological studies of ketamine in normal and NMDA epsilon1 receptor knockout mice. Br J Anaesth 92: 859-864.

Takahashi JS, DeCoursey PJ, Bauman L, Menaker M (1984). Spectral sensitivity of a novel photoreceptive system mediating entrainment of mammalian circadian rhythms. Nature 308: 186-188.
Trapani G, Altomare C, Liso G, Sanna E, Biggio G (2000). Propofol in anesthesia. Mechanism of action, structure-activity relationships, and drug delivery. Curr Med Chem 7: 249-271.

Tung A, Mendelson WB (2004). Anesthesia and sleep. Sleep Med Re 8: 213-225.

Tung A, Szafran MJ, Bluhm B, Mendelson WB (2002). Sleep deprivation potentiates the onset and duration of loss of righting reflex induced by propofol and isoflurane. Anesthesiology 97: 906-911.

Turek FW, Pinto LH, Vitaterna MH, Penev PD, Zee PC, Takahashi JS (1995). Pharmacological and genetic approaches for the study of circadian rhythms in mammals. Front Neuroendocrinol 16: 191-223.

Van Reeth O, Hinch D, Tecco JM, Turek FW (1991). The effects of short periods of immobilization on the hamster circadian clock. Brain Res 545: 208-214.

Wu CL, Berenholtz SM, Pronovost PJ, Fleisher LA (2002). Systematic review and analysis of postdischarge symptoms after outpatient surgery. Anesthesiology 96: 994-1003. 